\title{
Detecting an Itinerant Optical Photon Twice without Destroying It
}

\author{
Emanuele Distante $\odot$, Severin Daiss $\odot$, Stefan Langenfeld๑, Lukas Hartung, Philip Thomas $\odot$, \\ Olivier Morin $\odot$, Gerhard Rempe $\odot$, and Stephan Welte \\ Max-Planck-Institut für Quantenoptik, Hans-Kopfermann-Strasse 1, 85748 Garching, Germany
}

(Received 19 March 2021; accepted 18 May 2021; published 25 June 2021)

\begin{abstract}
Nondestructive quantum measurements are central for quantum physics applications ranging from quantum sensing to quantum computing and quantum communication. Employing the toolbox of cavity quantum electrodynamics, we here concatenate two identical nondestructive photon detectors to repeatedly detect and track a single photon propagating through a $60 \mathrm{~m}$ long optical fiber. By demonstrating that the combined signal-to-noise ratio of the two detectors surpasses each single one by about 2 orders of magnitude, we experimentally verify a key practical benefit of cascaded nondemolition detectors compared to conventional absorbing devices.
\end{abstract}

DOI: 10.1103/PhysRevLett.126.253603

Quantum physics distinguishes between two kinds of measurements. Following Pauli [1], a measurement of the first kind projects the state of a system onto an eigenstate of the measured observable, with subsequent measurements of the same kind giving the same result. A measurement of the second kind, in contrast, exerts a random backaction on the complementary observable so that repeated measurements lead to different results. This distinction has been refined by introducing the concept of a quantum nondemolition (QND) measurement [2,3], where Pauli's measurement of the first kind is formally defined by requesting that the operator corresponding to the measured observable commutes with the Hamiltonian of the system. In practice, QND measurements thus allow for repeated observations without changing the outcome, a unique property that has (at least) two benefits. First, it allows us to track the evolution of the observable without any backaction. Second, it allows us to concatenate several measurements, each with a nonperfect detection sensitivity (e.g., signal-tonoise ratio), and in this way enhance the overall sensitivity. Repeatability therefore has been identified as being a powerful advantage, as was emphasized by Caves et al. [4]: "The key feature of such a nondemolition measurement is repeatability —once is not enough!"

The application potential of QND measurements was realized early on in the field of gravitational wave detection $[2,3]$ and has later on sparked large interest in a vast number of other fields ranging from astronomy [5], to high-precision

Published by the American Physical Society under the terms of the Creative Commons Attribution 4.0 International license. Further distribution of this work must maintain attribution to the author(s) and the published article's title, journal citation, and DOI. Open access publication funded by the Max Planck Society. metrology [6,7] and quantum-information processing [8]. In the laboratory, QND measurements have been implemented in different matter-based experimental platforms such as ions [9], superconducting qubits [10], solid state systems [11], and atomic ensembles [12]. QND measurements of single photons, however, turned out to be comparatively difficult to implement, as they require the development of detectors capable of observing single photons without absorbing them [13]. Nevertheless, landmark experiments were proposed and performed in the microwave domain with photons stored in high-quality resonators [14-16] and later extended to the detection of itinerant microwave photons $[17,18]$. In the optical domain $[19,20]$, a single nondestructive detection of a flying photon was achieved as well [21]. Although this experiment demonstrated the principle of one QND detection, so far an experimental verification of the feasibility to repeatedly measure and thereby track a flying optical photon remained elusive.

Here, we verify the repeatability and the increased sensitivity by using two QND detectors to observe one optical photon twice. The two devices, each made from a single atom coupled to an optical cavity, are distributed along an optical fiber in which the photon propagates. Once the latter has interacted with each detector, we observe correlations between the detection events. These correlations are the key element behind our demonstration that the combined system of two detectors outperforms both individual devices in terms of signal-to-noise ratio. Furthermore, we demonstrate that our QND detectors are specifically suited to detect single-photon states. To this end, we employ the first QND detector as a state preparation device to generate single photons out of weak coherent states [22]. We then use such single photons to probe the second QND detector. As they are eigenstates of the measurement, we show that they are detected with higher probability than the input coherent states and that 
their single-photon character is unaffected by the QND detection. Our protocol is widely applicable and could also be implemented with single ions [23,24], superconducting qubits [17,18], quantum dots [25-27], silicon vacancy centers [28], or rare-earth ions [29] coupled to cavities.

The setup consists of a concatenation of QND detectors, lined up along an optical fiber in which a photon propagates. An artist's view of the experiment is shown in Fig. 1(a) where the QND detectors are depicted as bulbs that light up as soon as the photon passes by. An external observer can see correlations between the successively illuminated bulbs, and photon loss manifests by dark bulbs downstream. Figure 1(b) shows a more detailed sketch of our experiment. The two detectors, named QND1 and QND2 in the following, are separated by a distance of $60 \mathrm{~m}$. Each detector is made of a single ${ }^{87} \mathrm{Rb}$ atom trapped in a high-finesse cavity. The systems are both single-sided and operate in the strong-coupling regime of cavity quantum electrodynamics. The atoms are initialized in $\left|\uparrow_{z}\right\rangle=$ $\left|5^{2} S_{1 / 2}, F=2, m_{F}=2\right\rangle$ via optical pumping with light resonant with the $\left|\uparrow_{z}\right\rangle \leftrightarrow|e\rangle$ transition, where $|e\rangle=\left|5^{2} P_{3 / 2}, F=3, m_{F}=3\right\rangle$. The cavities are both stabilized to this transition frequency. Following the optical
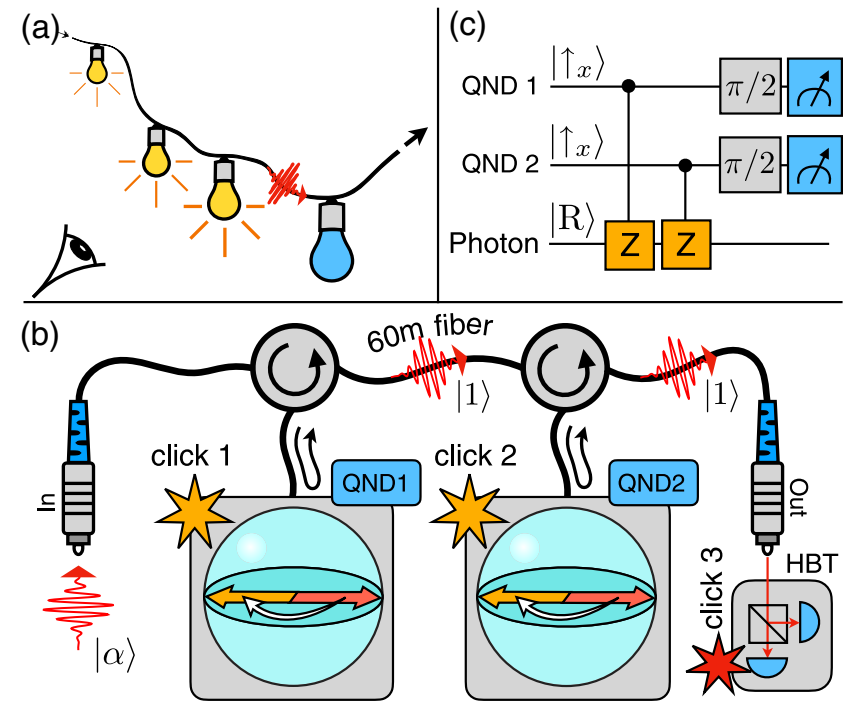

FIG. 1. (a) Our vision. A series of QND detectors, depicted as bulbs, are attached to an optical fiber. A propagating photon (red wiggly arrow) is subsequently detected several times, indicated by the illuminated bulbs. (b) Setup of the experiment. A weak coherent pulse $|\alpha\rangle$ is coupled into an optical fiber and consecutively interacts with the two QND detectors. The latter are composed of an atomic qubit each, depicted as a Bloch sphere (light blue). The north and south poles of the Bloch spheres correspond to $\left|\uparrow_{z}\right\rangle$ and $\left|\downarrow_{z}\right\rangle$, respectively. The states $\left|\uparrow_{x}\right\rangle\left(\left|\downarrow_{x}\right\rangle\right)$ on the equator are indicated by the red (yellow) arrows. Two circulators direct the photons onto the QND detectors before they are directed to a Hanbury Brown-Twiss (HBT) setup of singlephoton detectors (blue half disks). (c) Quantum circuit diagram. The photon reflections from the two detectors comprise two $Z$ gates. pumping, we employ a pair of Raman lasers in each of the setups to prepare both atoms in the superposition state $\left|\uparrow_{x}\right\rangle=(1 / \sqrt{2})\left(\left|\uparrow_{z}\right\rangle+\left|\downarrow_{z}\right\rangle\right)$, where $\left|\downarrow_{z}\right\rangle=\mid 5^{2} S_{1 / 2}, F=1$, $\left.m_{F}=1\right\rangle$ [21]. A state-detection laser resonant with the $\left|\uparrow_{z}\right\rangle \leftrightarrow|e\rangle$ transition allows us to deterministically distinguish between the states $\left|\uparrow_{z}\right\rangle$ and $\left|\downarrow_{z}\right\rangle$ with a fidelity of $>99 \%$.

Instead of injecting single photons into the fiber, we perform our experiments with weak coherent laser pulses $|\alpha\rangle$ that contain a mean photon number $|\alpha|^{2}=\langle n\rangle$ in front of the first QND detector. The choice of coherent pulses $(\lambda=780 \mathrm{~nm})$ allows us to study the application of our QND detectors as quantum state preparation devices of single photons. Additionally, in the limit of $\langle n\rangle \rightarrow 0$, we can approximate a single-photon input by eliminating the vacuum contribution through a post selection on a detection event ("click") in a standard absorbing detector at the far end of the propagation line. The light is injected into the fiber and, after the reflection from the QND detectors, hits two absorbing detectors arranged in Hanbury Brown-Twiss configuration that allows us to measure the second-order intensity autocorrelation function $g^{(2)}(\tau)$. The light pulses have a Gaussian shape with a full width at half maximum of $1 \mu \mathrm{s}$ and are resonant with the transition $\left|\uparrow_{z}\right\rangle \leftrightarrow|e\rangle$.

Our protocol, depicted as a quantum circuit diagram in Fig. 1(c), starts by initializing each atom in the state $\left|\uparrow_{x}\right\rangle$. As a next step, light injected into the fiber successively interacts with the two QND detectors. In case of an odd number of photons in the light pulse, as for a single photon, a phase shift of $\pi$ in the combined atom-light state leads to a sign change in both atomic superposition states [21,30-32]. Each of the QND detectors then occupies the state $\left|\downarrow_{x}\right\rangle=(1 / \sqrt{2})\left(\left|\uparrow_{z}\right\rangle-\left|\downarrow_{z}\right\rangle\right)$. For an even number of photons, such as for the vacuum state, the two atoms remain in $\left|\uparrow_{x}\right\rangle$. In the quantum-information language, the interaction of the photon and each of the atoms can be expressed as a controlled- $Z$ gate. After reflection of the light from the two detectors, a $\pi / 2$ pulse is applied which maps $\left|\downarrow_{x}\right\rangle\left(\left|\uparrow_{x}\right\rangle\right)$ onto $\left|\uparrow_{z}\right\rangle\left(\left|\downarrow_{z}\right\rangle\right)$. Therefore, a final atomic state detection of $\left|\uparrow_{z}\right\rangle\left(\left|\downarrow_{z}\right\rangle\right)$ heralds the presence of an odd (even) photon number at the corresponding detector.

Since the passing photon is ideally detected by both QND detectors, correlations between both of them must be observable. However in practice, the setup exhibits substantial losses between the two detectors (optical fiber coupling, fiber losses, and limited circulator transmission, total transmission: 53\%). To ensure the presence of light in front of each cavity, we can condition our data on the successful transmission of the photon through the entire system by postselecting on a click of one of our absorbing detectors downstream.

In a first experiment, we individually characterize the two QND detectors by measuring the probability of a successful QND photon detection conditioned on a click in the absorbing detectors. For the measurement, the mean 
photon number $\langle n\rangle$ in the impinging coherent pulse is scanned. For $\langle n\rangle=0.084$, the maximum probabilities $P\left(\uparrow_{z, \mathrm{QND} 1} \mid\right.$ click $)=81.3 \%$ and $P\left(\uparrow_{z, \mathrm{QND} 2} \mid\right.$ click $)=87.0 \%$ are observed. The difference in the measured maximum probabilities $P\left(\uparrow_{z, \mathrm{QND} 1 / 2} \mid\right.$ click $)$ is due to slightly different experimental parameters, mainly coherence time and the quality of the Raman pulses, in the two QND detectors. A detailed description of the individual characterization is given in the Supplemental Material [33].

In the next experiment, we concatenate the QND devices and remove the conditioning on the classical detector click. In a first step, we measure the click probabilities $P\left(\uparrow_{z, \mathrm{QND}} / 2\right)$ of the two detectors when probing them with a coherent state containing a mean photon number $\langle n\rangle$. Since the detectors are sensitive to the parity of the photon number, the respective click probability monotonically increases from zero to $50 \%$ as $\langle n\rangle$ is increased starting from zero. The saturation behavior results from the equal contributions of even and odd photon numbers in the limit of high $\langle n\rangle$. Because of the optical losses between the two setups, the respective mean photon number is different in front of the two detectors resulting in the observed scaling of the respective curves. Data are shown in Fig. 2.

Since the QND detectors are nondestructive, we can condition each of them on a click in the other detector. The respective click probabilities $P\left(\uparrow_{z, \mathrm{QND} 1 / 2} \mid \uparrow_{z, \mathrm{QND} 2 / 1}\right)$ show a different behavior compared to $P\left(\uparrow_{z, \mathrm{QND} 1 / 2}\right)$. When conditioning the downstream detector on the upstream detector, an increase in the click probability is observable compared to the case where no conditioning is applied. The effect,

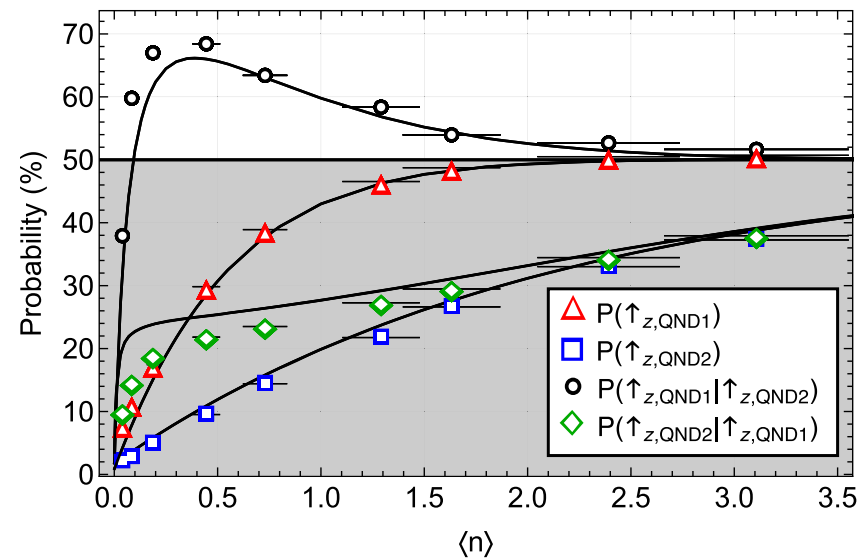

FIG. 2. Click probabilities $\mathrm{P}\left(\uparrow_{z, \mathrm{QND} 1}\right)$ (red triangles) and $\mathrm{P}\left(\uparrow_{z, \mathrm{QND} 2}\right)$ (blue squares) of the two QND detectors when probing them with a coherent state input. When conditioning on a click of the other detector respectively, we observe $\mathrm{P}\left(\uparrow_{z, \mathrm{QND} 1} \mid \uparrow_{z, \mathrm{QND} 2}\right)$ (black circles) and $\mathrm{P}\left(\uparrow_{z, \mathrm{QND} 2} \mid \uparrow_{z, \mathrm{QND} 1}\right)$ (green diamonds). The solid horizontal line shows the threshold of $50 \%$. The solid black lines are based on a simulation model outlined in the Supplemental Material [33]. The theory is not a fit, but employs experimental parameters characterized in independent measurements. The error bars represent standard deviations from the mean. however, is strongly suppressed due to the inevitable losses between the setups. In the other case, where the upstream detector is conditioned on the downstream detector, the effect of the losses is suppressed as, for $\langle n\rangle \ll 1$, a click downstream selects the events when no losses occurred between the setups. For increasing $\langle n\rangle$, we observe a correlation maximum of $P\left(\uparrow_{z, \mathrm{QND} 1} \mid \uparrow_{z, \mathrm{QND} 2}\right)=$ $(68.4 \pm 0.7) \%$, clearly surpassing the $50 \%$ threshold for uncorrelated random click events. For $\langle n\rangle$ approaching zero, these correlations decrease due to intrinsic dark counts in the QND detectors. The dark counts of QND1(2) stem from imperfect Raman rotations that leave $\mathrm{dc}_{1(2)}=1.4 \%(0.4 \%)$ residual population in the state $\left|\uparrow_{z}\right\rangle$ when performing two consecutive $\pi / 2$ pulses after initializing the atoms in $\left|\uparrow_{z}\right\rangle$. Because of higher photon-number contributions for increasing $\langle n\rangle$, the correlations asymptotically approach $50 \%$.

A benefit of nondestructive photon detectors compared to conventional destructive devices is that they can be concatenated to enhance the overall detection efficiency or the signal-to-noise ratio (SNR). In the following, we show that introducing a logical "or" $(\mathrm{V})$ connection between the QND detector clicks allows us to enhance the overall efficiency while a logical "and" $(\wedge)$ connection increases the signal-to-noise ratio. We start with the first case and measure the probability that at least one detector, QND1 or QND2, detects a photon conditioned on an absorbingdetector click downstream. A maximum probability of 95.1\% is observed, which surpasses the maximal capabilities of both individual QND devices (81.3\% and 87.0\%) and therefore shows the enhancement of the detection efficiency. Data as a function of $\langle n\rangle$ are shown in Fig. 3.

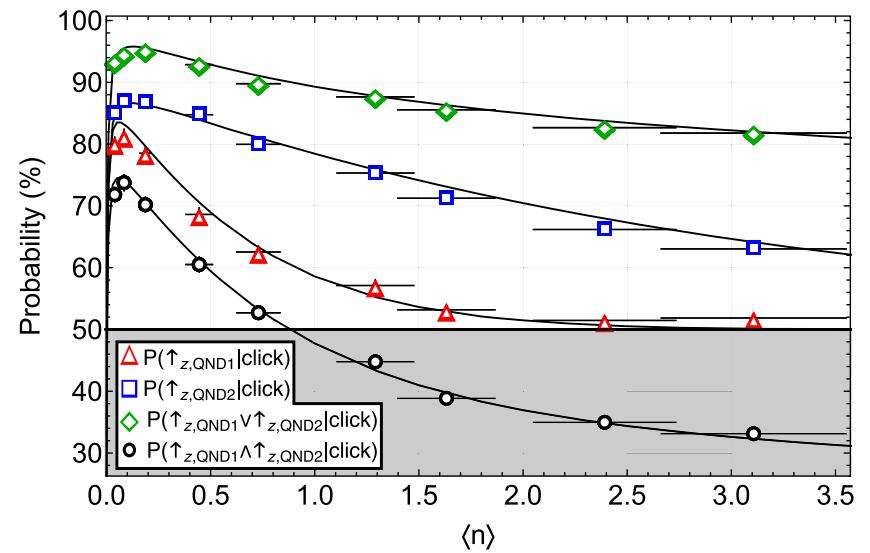

FIG. 3. Correlations between the QND detectors with conditioning on the absorbing-detector click downstream. The green diamonds and black circles, show the probability that QND1 or/ and QND2 clicks, conditioned on the absorbing-detector click. The black data points relate to Fig. 1(a), where ideally all upstream detectors click once the photon has passed. For comparison, we also show $P\left(\uparrow_{z, \mathrm{QND} 1 / 2} \mid\right.$ click) (red triangles and blue squares). Error bars represent standard deviations from the mean. The solid lines show the predictions based on our simulation model. 
A summary of the total measurement time, number of experimental runs, and coincidence rates is given in the Supplemental Material [33].

Although the efficiency is an important parameter of a single-photon detector, the signal-to-noise ratio is even more relevant in practice. In the limit of small $\langle n\rangle$, we define the SNR of the individual detectors as $\mathrm{SNR}_{1(2)}=$ $P\left(\uparrow_{z, \mathrm{QND1}(2)} \mid\right.$ click $) / \mathrm{dc}_{1(2)}$ and the SNR of the concatenated detectors as $\mathrm{SNR}_{1 \wedge 2}=P\left(\uparrow_{z, \mathrm{QND} 1} \wedge \uparrow_{z, \mathrm{QND} 2} \mid\right.$ click $) / \mathrm{dc}_{1 \wedge 2}$. In the last expression, $\mathrm{dc}_{1 \wedge 2}$ is the probability of finding both QND1 and QND2 in $\left|\uparrow_{z}\right\rangle$ at the end of the protocol when no light is injected into the fiber. By employing the logical "and" connection, we exploit the fact that the signal in both detectors is correlated while the dark counts are uncorrelated to enhance $\mathrm{SNR}_{1 \wedge 2}$ compared to $\mathrm{SNR}_{1}=59$ and $\mathrm{SNR}_{2}=218$. While in the limit of small $\langle n\rangle$ the probability $P\left(\uparrow_{z, \mathrm{QND} 1} \wedge \uparrow_{z, \mathrm{QND} 2} \mid\right.$ click $)$ is only slightly lowered compared to the individual devices (see Fig. 3), we find that $\mathrm{SNR}_{1 \wedge 2}$ is a factor of 61 higher than $\mathrm{SNR}_{2}$ and a factor of 227 higher than $\mathrm{SNR}_{1}$.

We now demonstrate that increasing the state overlap of the impinging light $|\alpha\rangle$ with that of a single-photon Fock state, $|1\rangle$, increases the click probability of QND2. This increase stems from the fact that single-photon states are eigenstates of our detector which are observed with unity efficiency in an ideal scenario. Starting with a weak coherent state at the input of the fiber, we employ two conditions ensuring that the light impinging on QND2 is approximately described by a single-photon state. First, the condition on a click in the downstream absorbing detectors is applied. This condition removes the vacuum contribution from the coherent state and additionally ensures that light is not lost in the fiber and therefore impinges on QND2. The limitations of this condition are that all photon-number contributions $n \geq 1$ will remain in the pulse, and that for too low values of $\langle n\rangle$ the dark counts in the absorbing detectors add a small vacuum contribution. Nevertheless, for appropriately chosen low values of $\langle n\rangle$, this technique allows us to approximate single photons. Second, and as an additional condition, we employ QND1 as a state preparation device. Since it is sensitive to the parity of the impinging photon number [38,39], a click in this detector removes all even photon-number contributions [22]. Therefore, in the limit of vanishing $\langle n\rangle$, employing both conditions applies a selection window around the desired single-photon Fock state and approximates a single photon better than just conditioning on the absorbing detector. As a result, for a proper single-photon detector, the following inequality must hold: $P\left(\uparrow_{z, \mathrm{QND} 2} \mid \uparrow_{z, \mathrm{QND} 1} \wedge\right.$ click $)>$ $P\left(\uparrow_{z, \mathrm{QND} 2} \mid\right.$ click $)$. As shown in Fig. 4 , we can indeed verify this inequality. Our data show that the click probability of QND2 increases if the impinging state has a higher overlap with an ideal single-photon state. Maximally, a probability $P\left(\uparrow_{z, \mathrm{QND} 2} \mid \uparrow_{z, \mathrm{QND} 1} \wedge\right.$ click $)=$ $90.7 \%$ is observed.

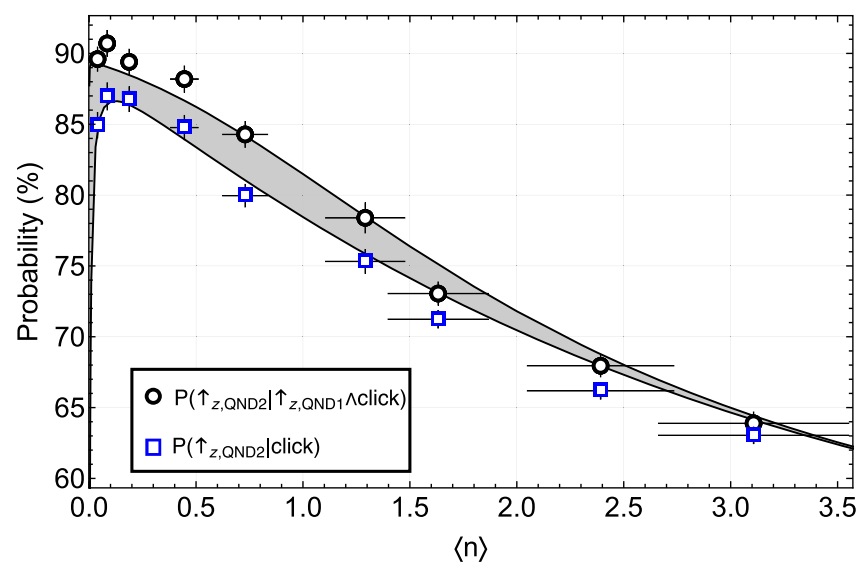

FIG. 4. Probabilities $P\left(\uparrow_{z, \mathrm{QND} 2} \mid \uparrow_{z, \mathrm{QND} 1} \wedge\right.$ click) (black circles) and $P\left(\uparrow_{z, \mathrm{QND} 2} \mid\right.$ click) (blue squares). The solid lines show theoretical predictions based on our model. The gray area highlights the difference between the two theoretical curves. Error bars represent standard deviations from the mean.

To verify the preparation of single photons with our two QND detectors from the initial coherent pulse, we employ two absorbing detectors in a Hanbury Brown-Twiss configuration as shown in Fig. 1(b). This allows us to extract the second-order photon-correlation function $g^{(2)}(\tau)$. For the measurement, we use an initial coherent pulse with an average photon number of $\langle n\rangle=0.45$. We extract $g^{(2)}(0)$ and $\bar{g}^{(2)}(\tau \neq 0)$ averaged over all $\tau \neq 0$. The second-order correlation function is conditioned on clicks in the two QND measurements. Table I contains the obtained data. When not conditioning on any of the QND detection results, we verify the coherent character of our light since $g^{(2)}(\tau)$ is close to unity for all values of $\tau$. As a next step, we condition our data on a click of QND1 and observe a reduction of $g^{(2)}(0)$ from unity to $0.354_{-0.091}^{+0.121}$. Similarly, when conditioning the data on a click of QND2, we obtain $g^{(2)}(0)=0.047_{-0.015}^{+0.021}$. Both results show the single-photon character of the light after conditioning on the respective detector. The difference in the two obtained values for $g^{(2)}(0)$ stems from the different mean photon number in the coherent pulse impinging onto the respective QND detector

TABLE I. Measurements of the second-order photoncorrelation function $g^{(2)}(\tau)$. The obtained data is conditioned on different combinations of QND detection events. Error bars are statistical.

\begin{tabular}{lll}
\hline \hline Condition & $g^{(2)}(\tau=0)$ & $\bar{g}^{(2)}(\tau \neq 0)$ \\
\hline None & $1.005_{-0.206}^{+0.253}$ & $0.995_{-0.005}^{+0.006}$ \\
$\uparrow_{z, \text { QND1 }}$ & $0.354_{-0.091}^{+0.121}$ & $1.010_{-0.004}^{+0.004}$ \\
$\uparrow_{z, \text { QND2 }}$ & $0.047_{-0.015}^{+0.021}$ & $1.000_{-0.002}^{+0.002}$ \\
$\uparrow_{z, \text { QND1 }} \wedge \uparrow_{z, \text { QND2 }}$ & $0.038_{-0.014}^{+0.023}$ & $1.000_{-0.002}^{+0.002}$ \\
\hline \hline
\end{tabular}


due to the losses between the detectors. Finally, when the data is conditioned on a click of both QND detectors, we obtain $g^{(2)}(0)=0.038_{-0.014}^{+0.023}$. A comparison between the conditions $\uparrow_{z, \mathrm{QND} 1}$ and $\uparrow_{z, \mathrm{QND} 1} \wedge \uparrow_{z, \mathrm{QND} 2}$ shows that the single-photon character of the light after successful state preparation with QND1 is preserved by QND2.

In conclusion, we have shown the feasibility of repeatedly detecting an optical photon. This feature of a QND measurement allows us to enhance the detection efficiency or the signal-to-noise ratio. By adding time resolution to the QND detector, it should be possible to gain information about the propagation direction of a photon, a piece of information only accessible with QND detectors. Moreover, an improved setup with smaller losses between the two detectors could serve as a heralded source of photonic Fock states. Such a device, described in detail in the Supplemental Material [33], is capable of decomposing a coherent state into its number-state constituents [16]. Arguably most fascinating is an extension to several nondestructive detectors for photonic qubits. This could speed up a plethora of quantum-network [40-42] protocols such as entanglement distribution where a new transmission attempt could be started immediately after a spatially resolved loss detection between sender and receiver [43].

This work was supported by the Bundesministerium für Bildung und Forschung via the Verbund Q.Link.X (16KIS0870), by the Deutsche Forschungsgemeinschaft under Germany's Excellence Strategy-EXC-2111390814868, and by the European Union's Horizon 2020 research and innovation programme via the project Quantum Internet Alliance (QIA, GA No. 820445). E. D. acknowledges support by the Cellex-ICFO-MPQ postdoctoral fellowship program in the early stages of the experiment.

*Corresponding author.

stephan.welte@mpq.mpg.de

[1] W. Pauli, in Die Allgemeinen Prinzipien der Wellenmechanik, Handbuch der Physik, 2. Auflage. Bd. 24, 1. Teil. S. (Springer, Berlin, 1933), pp. 83-272.

[2] V. B. Braginsky, Y. I. Vorontsov, and K. S. Thorne, Quantum nondemolition measurements, Science 209, 547 (1980).

[3] V. B. Braginsky and F. Ya. Khalili, Quantum nondemolition measurements: The route from toys to tools, Rev. Mod. Phys. 68, 1 (1996).

[4] C. M. Caves, K. S. Thorne, R. W. P. Drever, V. D. Sandberg, and M. Zimmermann, On the measurement of a weak classical force coupled to a quantum-mechanical oscillator. I. Issues of principle, Rev. Mod. Phys. 52, 341 (1980).

[5] A. Kellerer, Quantum telescopes, Astron. Geophys. 55, 3.28 (2014).

[6] V. Giovannetti, S. Lloyd, and L. Maccone, Quantumenhanced measurements: Beating the standard quantum limit, Science 306, 1330 (2004).
[7] J. Ma, X. Wang, C.P. Sun, and F. Nori, Quantum spin squeezing, Phys. Rep. 509, 89 (2011).

[8] T. C. Ralph, S. D. Bartlett, J. L. O’Brien, G. J. Pryde, and H. M. Wiseman, Quantum nondemolition measurements for quantum information, Phys. Rev. A 73, 012113 (2006).

[9] D. B. Hume, T. Rosenband, and D. J. Wineland, HighFidelity Adaptive Qubit Detection through Repetitive Quantum Nondemolition Measurements, Phys. Rev. Lett. 99, 120502 (2007).

[10] A. Lupaşcu, S. Saito, T. Picot, P. C. de Groot, C. J. P. M. Harmans, and J. E. Mooij, Quantum non-demolition measurement of a superconducting two-level system, Nat. Phys. 3, 119 (2007).

[11] P. Neumann, J. Beck, M. Steiner, F. Rempp, H. Fedder, P. R. Hemmer, J. Wrachtrup, and F. Jelezko, Single-shot readout of a single nuclear spin, Science 329, 542 (2010).

[12] A. Kuzmich, L. Mandel, and N. P. Bigelow, Generation of Spin Squeezing Via Continuous Quantum Nondemolition Measurement, Phys. Rev. Lett. 85, 1594 (2000).

[13] V. B. Braginsky, Quantum-non-demolition measurements of the energy of standing and flying photons, in Proceedings of the 3rd International Symposium on Foundations of Quantum Mechanics, Tokyo (1989), pp. 135-139, https://dcc.ligo .org/LIGO-T890016/public.

[14] M. Brune, S. Haroche, J. M. Raimond, L. Davidovich, and N. Zagury, Manipulation of photons in a cavity by dispersive atom-field coupling: Quantum-nondemolition measurements and generation of "Schrödinger cat" states, Phys. Rev. A 45, 5193 (1992).

[15] G. Nogues, A. Rauschenbeutel, S. Osnaghi, M. Brune, J. M. Raimond, and S. Haroche, Seeing a single photon without destroying it, Nature (London) 400, 239 (1999).

[16] C. Guerlin, J. Bernu, S. Deléglise, C. Sayrin, S. Gleyzes, S. Kuhr, M. Brune, J. M. Raimond, and S. Haroche, Progressive field-state collapse and quantum non-demolition photon counting, Nature (London) 448, 889 (2007).

[17] S. Kono, K. Koshino, Y. Tabuchi, A. Noguchi, and Y. Nakamura, Quantum non-demolition detection of an itinerant microwave photon, Nat. Phys. 14, 546 (2018).

[18] J.-C. Besse, S. Gasparinetti, M. C. Collodo, T. Walter, P. Kurpiers, M. Pechal, C. Eichler, and A. Wallraff, SingleShot Quantum Nondemolition Detection of Individual Itinerant Microwave Photons, Phys. Rev. X 8, 021003 (2018).

[19] S. R. Friberg, S. Machida, and Y. Yamamoto, QuantumNon-Demolition Measurement of the Photon Number of an Optical Soliton, Phys. Rev. Lett. 69, 3165 (1992).

[20] P. Grangier, J. A. Levenson, and J.-P. Poizat, Quantum nondemolition measurements in optics, Nature (London) 396, 537 (1998).

[21] A. Reiserer, S. Ritter, and G. Rempe, Nondestructive detection of an optical photon, Science 342, 1349 (2013).

[22] S. Daiss, S. Welte, B. Hacker, L. Li, and G. Rempe, SinglePhoton Distillation Via a Photonic Parity Measurement Using Cavity QED, Phys. Rev. Lett. 122, 133603 (2019).

[23] A. B. Mundt, A. Kreuter, C. Becher, D. Leibfried, J. Eschner, F. Schmidt-Kaler, and R. Blatt, Coupling a Single Atomic Quantum Bit to a High Finesse Optical Cavity, Phys. Rev. Lett. 89, 103001 (2002). 
[24] H. Takahashi, E. Kassa, C. Christoforou, and M. Keller, Strong Coupling of a Single Ion to an Optical Cavity, Phys. Rev. Lett. 124, 013602 (2020).

[25] I. Fushman, D. Englund, A. Faraon, N. Stoltz, P. Petroff, and J. Vučković, Controlled phase shifts with a single quantum dot, Science 320, 769 (2008).

[26] H. Kim, R. Bose, T. S. Shen, G. S. Solomon, and E. Waks, A quantum logic gate between a solid-state quantum bit and a photon, Nat. Photonics 7, 373 (2013).

[27] L. De Santis, C. Antón, B. Reznychenko, N. Somaschi, G. Coppola, J. Senellart, C. Gómez, A. Lemaître, I. Sagnes, A. G. White, L. Lanco, A. Auffèves, and P. Senellart, A solid-state single-photon filter, Nat. Nanotechnol. 12, 663 (2017).

[28] M. K. Bhaskar, R. Riedinger, B. Machielse, D. S. Levonian, C. T. Nguyen, E. N. Knall, H. Park, D. Englund, M. Lončar, D. Sukachev, and M. D. Lukin, Experimental demonstration of memory-enhanced quantum communication, Nature (London) 580, 60 (2020).

[29] S. Chen, M. Raha, C. M. Phenicie, S. Ourari, and J. D. Thompson, Parallel single-shot measurement and coherent control of solid-state spins below the diffraction limit, Science 370, 592 (2020).

[30] L.-M. Duan and H. J. Kimble, Scalable Photonic Quantum Computation Through Cavity-Assisted Interactions, Phys. Rev. Lett. 92, 127902 (2004).

[31] Y.-F. Xiao, X.-M. Lin, J. Gao, Y. Yang, Z.-F. Han, and G.-C. Guo, Realizing quantum controlled phase flip through cavity QED, Phys. Rev. A 70, 042314 (2004).

[32] T. G. Tiecke, J. D. Thompson, N. P. de Leon, L. R. Liu, V. Vuletić, and M.D. Lukin, Nanophotonic quantum phase switch with a single atom, Nature (London) 508, 241 (2014).

[33] See Supplemental Material at http://link.aps.org/ supplemental/10.1103/PhysRevLett.126.253603 for a detailed description of the theoretical model, an individual characterization of the two QND detectors, and a proposal for a heralded source of photon number states, which contains Refs. [34-37].

[34] J. R. Johansson, P. D. Nation, and F. Nori, QuTiP: An opensource PYTHON framework for the dynamics of open quantum systems, Comput. Phys. Commun. 183, 1760 (2012).

[35] A. Kuhn, Cavity induced interfacing of atoms and light, in Engineering the Atom-Photon Interaction, edited by A. Predojević and M.W. Mitchell (Springer, Cham, 2015), pp. 3-38.

[36] W. Rosenfeld, F. Hocke, F. Henkel, M. Krug, J. Volz, M. Weber, and H. Weinfurter, Towards Long-Distance Atom-Photon Entanglement, Phys. Rev. Lett. 101, 260403 (2008).

[37] M. A. Nielsen and I. L. Chuang, Quantum Computation and Quantum Information (Cambridge University Press, Cambridge, England, 2000).

[38] B. Wang and L.-M. Duan, Engineering superpositions of coherent states in coherent optical pulses through cavityassisted interaction, Phys. Rev. A 72, 022320 (2005).

[39] B. Hacker, S. Welte, S. Daiss, A. Shaukat, S. Ritter, L. Li, and G. Rempe, Deterministic creation of entangled atomlight Schrödinger-cat states, Nat. Photonics 13, 110 (2019).

[40] H. J. Kimble, The quantum internet, Nature (London) 453, 1023 (2008).

[41] A. Reiserer and G. Rempe, Cavity-based quantum networks with single atoms and optical photons, Rev. Mod. Phys. 87, 1379 (2015).

[42] S. Wehner, D. Elkouss, and R. Hanson, Quantum internet: A vision for the road ahead, Science 362, eaam9288 (2018).

[43] D. Niemietz, P. Farrera, S. Langenfeld, and G. Rempe, Nondestructive detection of photonic qubits, Nature (London) 591, 570 (2021). 